$\mathrm{UW} / \mathrm{PT}-96-18$

DOE/ER/40561-285-INT96-00-145

RU-96-84

\title{
Massless Composite Fermions in Two Dimensions and the Overlap.
}

\author{
Rajamani Narayanan ${ }^{1}$ and Herbert Neuberger ${ }^{2}$ \\ ${ }^{1}$ Institute for Nuclear Theory, Box 351550 \\ University of Washington, Seattle, WA 98195-1550. \\ ${ }^{2}$ Department of Physics and Astronomy \\ Rutgers University, Piscataway, NJ 08855-0849.
}

\begin{abstract}
There exist chiral gauge models in two dimensions that have massless composite fermions. Two examples are presented and it is suggested that they be accepted as benchmark test-cases for generic proposals of non-perturbatively regulating chiral gauge theories in any dimension. We apply the overlap to the simpler of the two benchmarks and present the results of a numerical simulation of modest size.
\end{abstract}


Chiral symmetries can ensure the masslessness of fermions; thus, chiral gauge theories might produce massless composite fermions. A major objective of lattice formulations of chiral gauge theories is to provide a tool to study this non-perturbative phenomenon numerically. Given a proposal, before jumping to costly simulations in four dimensions, one needs to establish confidence in the technique and a natural theoretical laboratory is offered by the two dimensional world. A proposal that fails in two dimensions is unlikely to be useful in four.

The possibility of composite massless fermions was intensely studied in the late seventies and early eighties [1]. The major ideas in this context were the maximal attractive channel (MAC) hypothesis, complementarity between the Higgs and the confining phase descriptions and fulfillment of 't Hooft's consistency conditions [2]. In search of a dynamically controlled environment where these ideas could be tested and applied the two dimensional laboratory was visited already at that time, but the results were somewhat disappointing [3].

Today, when we again turn to two dimensions, we must look for models that, not withstanding the earlier work, nevertheless provide interesting test-cases for numerical techniques. Our first example has already been put to use by us before [4], but its major dynamical features that single it out for our purposes were not described.

The model ("11112") contains four left-handed Weyl fermions (LW) carrying a $U(1)$ charge 1 and one right-handed Weyl fermion (RW) of charge 2. Anomalies cancel for this $U(1)$ and it can be gauged. The model also has an anomalous global $U(4)$ symmetry. Consider first the $S U(4)(\operatorname{Spin}(6))$ component: The constituent anomaly would match with composites making up a six dimensional real representation (second rank antisymmetric in $S U(4)$ language) by left-handed Majorana-Weyl (LMW) fermions. If there are no other massless particles gravitational anomalies also match. The $U(1)$ component of the global $U(4)$ is broken explicitly by instantons in the gauged $U(1)$, so there is no matching condition associated with it. These kinematic considerations are supported by the exact solution of the model:

The action of the 11112 model in Euclidean space is:

$$
S=\frac{1}{4 e_{0}^{2}} \int d^{2} x F_{\mu \nu}^{2}-\sum_{k=1}^{4} \int d^{2} x \bar{\chi}_{k} \sigma_{\mu}\left(\partial_{\mu}+i A_{\mu}\right) \chi_{k}-\int d^{2} x \bar{\psi} \sigma_{\mu}^{*}\left(\partial_{\mu}+2 i A_{\mu}\right) \psi
$$

where $\sigma_{1}=1$ and $\sigma_{2}=i$. The candidate interpolating fields for the composites are:

$$
\eta_{i j}=\chi_{i} \chi_{j} \bar{\psi}, \quad \bar{\eta}_{i j}=\bar{\chi}_{i} \bar{\chi}_{j} \psi
$$

As long as the gauge field $A_{\mu} \equiv \partial_{\mu} \Phi+\epsilon_{\mu \nu} \partial_{\nu} \phi$ carries zero topological charge the path integral can be done by changing variables: $\chi_{i}=\chi_{i}^{0} e^{-i \Phi-\phi}, \bar{\chi}_{i}=\bar{\chi}_{i}^{0} e^{i \Phi+\phi}, \psi=\psi^{0} e^{-2 i \Phi+2 \phi}$, 
$\bar{\psi}=\bar{\psi}^{0} e^{2 i \Phi-2 \phi}$. All fields with a zero superscript have a free field action. The transformation has a Jacobian which together with the original pure gauge term gives $S_{g}=$ $\frac{1}{2 e_{0}^{2}} \int d^{2} x\left(\partial^{2} \phi\right)^{2}-\frac{2}{\pi} \int d^{2} x \phi \partial^{2} \phi$. Thus the path integral is quadratic at each stage [5]. The disappearance of $\Phi$ reflects anomaly cancelation for the gauged $U(1)$.

We first evaluate the fermion number conserving two point function of the composites:

$$
<\eta_{i j}(x) \bar{\eta}_{k l}(y)>=\left(\delta_{i k} \delta_{j l}-\delta_{i l} \delta_{j k}\right) \frac{1}{(2 \pi)^{3}} \frac{1}{(\sigma \cdot z)^{2}} \frac{1}{\sigma^{*} \cdot z} e^{8<[\phi(x)-\phi(y)]^{2}>}
$$

where $z=x-y$. Using

$$
<\phi(x) \phi(y)>-<\phi^{2}(0)>=-\frac{1}{16}\left[\int_{0}^{\infty} \frac{d t}{t} e^{-\frac{1}{t}-\frac{t e_{0}^{2} z^{2}}{\pi}}+2 \gamma+\log \frac{e_{0}^{2} z^{2}}{\pi}\right] \equiv-\frac{1}{16} G\left(z^{2}\right)
$$

we get

$$
e^{8<[\phi(x)-\phi(y)]^{2}>}=e^{G\left(z^{2}\right)} .
$$

As $z^{2} \rightarrow \infty$, we obtain:

$$
<\eta_{i j}(x) \bar{\eta}_{k l}(y)>\sim\left(\delta_{i k} \delta_{j l}-\delta_{i l} \delta_{j k}\right) \frac{e_{0}^{2}}{4 \pi^{3}} e^{2 \gamma} \frac{1}{2 \pi \sigma \cdot z}\left[1+\mathcal{O}\left(e^{-\frac{2 e_{0}|z|}{\sqrt{\pi}}}\right)\right]
$$

Normalizing the interpolating fields $\eta$ we see that we have a massless pole corresponding to a left-mover. Note that the corrections are exponential, so no couplings to other massless particles are evident (absence of cuts).

To ascertain that we have indeed LMW particles and not LW particles we need to also compute the $\langle\eta \eta>$ and $\langle\bar{\eta} \bar{\eta}>$ correlation functions. These propagators don't vanish since fermion number conservation is broken by instanton effects. More precisely, single instantons allow violations by two units, so indeed the particle and anti-particle created by an $\eta$ can mix. One cannot calculate the new propagators by the above method directly since nontrivial topology is needed. However, using clustering, the infinite volume computation (which is all we are doing) can be replaced by a calculation at trivial topology. We start from $<\eta_{12}(x) \eta_{34}(x+\Delta) \bar{\eta}_{12}(y) \bar{\eta}_{34}(y+\Delta)>$. The computation proceeds as before and an exact expression is obtained:

$$
\begin{gathered}
<\eta_{12}(x) \eta_{34}(x+\Delta) \bar{\eta}_{12}(y) \bar{\eta}_{34}(y+\Delta)>= \\
\frac{1}{(2 \pi)^{6}} \frac{1}{(\sigma \cdot z)^{4}} \frac{\left(\sigma^{*} \cdot \Delta\right)^{2}}{\left(\sigma^{*} \cdot z\right)^{2}\left[\left(\sigma^{*} \cdot z\right)^{2}-\left(\sigma^{*} \cdot \Delta\right)^{2}\right]} e^{-2 G\left(\Delta^{2}\right)+G\left(|z+\Delta|^{2}\right)+G\left(|z-\Delta|^{2}\right)+2 G\left(z^{2}\right) .}
\end{gathered}
$$

We take now the separation $z=x-y$ to infinity and, asserting that clustering holds obtain:

$$
<\eta_{12}(x) \eta_{34}(x+\Delta)><\bar{\eta}_{12}(y) \bar{\eta}_{34}(y+\Delta)>=\frac{1}{(2 \pi)^{6}} e^{-2 G\left(\Delta^{2}\right)} e^{8 \gamma}\left(\frac{e_{0}^{2}}{\pi}\right)^{4}\left(\sigma^{*} \cdot \Delta\right)^{2} .
$$


Using translational invariance, Lorentz invariance, and charge conjugation, we end up having to take a square root obtaining:

$$
<\eta_{12}(0) \eta_{34}(\Delta)>=<\bar{\eta}_{12}(0) \bar{\eta}_{34}(\Delta)>\sim \frac{1}{(2 \pi)^{3}} e^{-G\left(\Delta^{2}\right)} e^{4 \gamma}\left(\frac{e_{0}^{2}}{\pi}\right)^{2}\left(\sigma^{*} \cdot \Delta\right) .
$$

The undetermined overall signs for all index combinations can be absorbed in three of the six $\eta$-fields and we shall assume this has been done.

From the above equation we extract both the asymptotics as $|\Delta| \rightarrow \infty$ and as $|\Delta| \rightarrow 0$. Going to large separation we get

$$
<\eta_{i j}(x) \eta_{k l}(y)>=<\bar{\eta}_{i j}(x) \bar{\eta}_{k l}(y)>\sim-\epsilon_{i j k l} \frac{e_{0}^{2}}{4 \pi^{3}} e^{2 \gamma} \frac{1}{2 \pi \sigma \cdot z}\left[1+\mathcal{O}\left(e^{-\frac{2 e_{0}|z|}{\sqrt{\pi}}}\right)\right] .
$$

The $\frac{e_{0}^{2}}{4 \pi^{3}} e^{2 \gamma}$ prefactor is the same as in the $\langle\eta \bar{\eta}>$ correlation function, so the same normalization makes the propagators canonical. Going to short distances we extract the expectation value of the leading operator:

$$
<\eta_{i j}(0)(\sigma \cdot \partial) \eta_{k l}(0)>=\epsilon_{i j k l} \frac{e_{0}^{4} e^{4 \gamma}}{4 \pi^{5}}
$$

Defining the dimensionless 't Hooft vertex $V=\frac{\pi^{2}}{e_{0}^{4}} \chi_{1} \chi_{2} \chi_{3} \chi_{4} \psi(\sigma \cdot \partial) \psi$ we obtain

$$
<V>=\frac{e^{4 \gamma}}{4 \pi^{3}} \approx 0.081
$$

The 't Hooft vertex has the form of a term in the kinetic energy for composite massless left-movers in a low energy effective Lagrangian. The above expectation value arises from a single instanton background plus small fluctuations around it. Other terms in the kinetic energy get an expectation value in zero topology. One cannot argue convincingly (in lieu of the exact solution) that the required "exponentiation" occurs since there is no justifiable "dilute gas approximation". However, if we adapt "complementarity" [2] to two dimensions we could say that we have an effective scalar field made out of, say, $\chi_{1} \bar{\psi}$ and its conjugate. This scalar field behaves as if it has a negative mass, but, in two dimensions, unlike in four, it does not condense [6]. This composite "Higgs" field in two dimensions affects the $U(1)$ gauge dynamics in a different way from four. Instead of making the forces short range it preserves confinement via vortex (instanton) formation. What changes as a result of the sign of the mass is only the size of the string tension. Now confinement is a semiclassical effect and the "dilute gas" picture can be made to hold. Within the dilute gas picture one can argue that the 't Hooft vertex exponentiates in the usual way. Of course, this is only a description which, while not really applicable, is hoped to lead to the correct conclusions 
regarding the low mass spectrum. The exponentiation leads us to an effective Lagrangian describing LMW particles.

Since we are in the fortunate position of having exact results we do not need to appeal to complementarity to argue that indeed only a sextet of LMW particles is massless. We define the linear combinations

$$
\rho_{i j}^{ \pm}=\frac{\pi^{\frac{3}{2}} e^{-\gamma}}{e_{0}}\left[\eta_{i j} \pm \frac{1}{2} \epsilon_{i j k l} \bar{\eta}_{k l}\right] .
$$

While

$$
<\rho_{i j}^{-}(x) \rho_{k l}^{-}(y)>\sim-\epsilon_{i j k l} \frac{1}{2 \pi \sigma \cdot z}
$$

correlators involving $\rho^{+}$have no massless poles, and no cuts extending to the origin. In short the massless spectrum consists of six LMW fermions.

Clearly, a numerical method could establish its credentials by reproducing the above gauge invariant spectrum. It is obvious that a scheme that does not "quite work" in the presence of nonzero topology would fail completely. We proceed now to a non-Abelian model where the issue of topology does not arise, and where a scheme could be tested even if it is known to mishandle topology. At present we have no exact results about the non-Abelian model, although we would not exclude the possibility that the spectrum of massless composites could be established by analytical means also in this case.

The gauge group is $S U(2)$ and matter consists of two LW doublets $\chi_{i \alpha}$ and one RMW triplet $\psi_{A} . \alpha, \beta=1,2, a=1,2,3$ refer to flavor (not gauged) and $i, j, k=1,2, A=1,2,3$ refer to color (gauged). The flavor symmetry group is $U(2)$ and there are no instantons.

Using $\tau$ to denote Pauli matrices we guess that the massless composites are given by (upper or lower positions of indices are meaningless) :

$$
\eta_{a}=\tau_{a}^{\alpha \beta} \tau_{A}^{i j} \bar{\chi}_{i \alpha} \chi_{j \beta} \psi_{A}, \quad \rho=\tau_{A}^{i j} \epsilon^{j k} \epsilon^{\alpha \beta} \chi_{i \alpha} \chi_{k \beta} \psi_{A}, \quad \bar{\rho}=\tau_{A}^{i j} \epsilon^{j k} \epsilon^{\alpha \beta} \bar{\chi}_{i \alpha} \bar{\chi}_{k \beta} \psi_{A}
$$

The $\eta$ 's are a flavor $S U(2)$ triplet made out of LMW particles and the $\rho$ is a flavor $S U(2)$ singlet LW particle. The $\eta$ 's carry no flavor $U(1)$ charge but $\rho$ is doubly charged. It is simple to check that the flavor $U(1), S U(2)$ and the gravitational anomalies match between the constituents and the composites.

The Abelian and non-Abelian models can be connected by adding a Higgs color triplet to the non-Abelian one. The absence of instantons makes it plausible that things look more like in four dimensions. Thus, we expect two of the three gauge bosons to become massive leaving us with a confining $U(1)$. Switching some particles with anti-particles we see that the $\chi$ fields here play the role of the $\chi$ fields in the Abelian case and the RW $\psi$ there is made up of $\psi_{1} \pm i \psi_{2}$ here. So, on the basis of the Abelian model we expect six $L M W$ particles and, in addition, the left over massless neutral RMW $\psi_{3}$. One can imagine that 
a Majorana mass-term connecting $\psi_{3}$ to one of the six left-movers gets generated and one has the same number of massless particles (five LMW) as in the analysis above.

Of course, we cannot be sure that we have correctly identified the massless spectrum in the non-Abelian case. But it is plausible that it is just as simple as we have guessed. So, if a simulation reproduced this spectrum one could feel encouraged to believe in the technique.

There is one issue that spoils the usefulness of two dimensions to some extent [7]. Four fermi interactions can be marginal or even marginally relevant in two dimensions. Typically, the symmetries of gauge theories do not exclude these terms, and a generic regularization will generate them. Their presence in the continuum limit can have dramatic effects. To usefully test a procedure on two dimensional benchmarks one would need to do some tuning to minimize the effective couplings of all possible four fermi terms. For example, in the Abelian model the following (Thirring) term is exactly marginal and allowed by all symmetries:

$$
\int d^{2} x \sum_{k}\left[\bar{\chi}_{k} \chi_{k}\right] \bar{\psi} \psi
$$

Its inclusion does not spoil the solubility of the model, but does do away with the simple pole structure in the infrared, replacing it by cuts. One no longer has particles and an $S$-matrix in the usual sense. This is the single four fermi term possible so tuning it away (it is only marginal - not relevant) is a nuisance one can hope to live with.

We now turn to applying the overlap prescription to the Abelian model. When facing a numerical simulation one needs to formulate the theory in some finite volume, typically a torus (since otherwise one has no remnant of translational invariance). With massless particles around this can be delicate but the issue has been settled for our case in our previous work [4]. It turns out that a good definition on a torus consists of making $\psi$ periodic in both directions (PP) and the four $\chi$ fields obey PP, AP, AA, PA boundary conditions respectively (A=anti-periodic). (Note that the global $S U(4)$ is broken by the boundary conditions although a cyclic four element symmetry coupled to rotations is preserved. Incidentally, this also singles out only one of the $\chi$ 's $(\mathrm{PP})$ as a possible partner of $\psi$ in the complementary, effective Higgs field picture).

Having settled the issue of boundary conditions one is still faced with the fact that one would need a finite large box to observe the power decay of the composites' correlation functions. Since the composites are not pointlike, one also needs to keep a few lattice points "inside" them. This is a typical numerical problem faced whenever one does Monte Carlo simulations. In our case, in view of the fact that the algorithm essentially involves the full computation of fermionic determinants, the resource requirements are comparable to those of a modern large scale numerical Monte Carlo computation in four dimensions. As a first step it makes sense to devise a smaller project, which can be still done on a 
single workstation in a reasonable amount of time. This was something we have been preparing for when we did a small scale simulation for the vector-like Schwinger model $[7,8]$. The objective is to measure $\langle V\rangle$, the 't Hooft vertex expectation value in small to moderate physical volumes. Although finite size effects are sizable, they are computable and seeing that one gets reasonable numbers is a strong indications that things would work out correctly in a large scale simulation also. On the other hand, if $\langle V\rangle$ does not come out right, it is very unlikely that the correct particle spectrum will be obtained in a large scale simulation. Moreover, the smaller project sets the correct scale, parameters and helps determining how to tune away the Thirring term.

Since the Thirring term will be also a nuisance in vector-like simulations, and the latter can be simulated within significantly less computer time, because one does not need to do numerical gauge averaging along orbits as gauge invariance is exact, we learn how to tune its coefficient in the vector-like case first. Of course, there is no guarantee that this will really apply to the chiral case. However, there does exist a vector-like theory that is quite close to our chiral model. It is the four flavor Schwinger model, defined by:

$$
S_{v}=\frac{1}{4 e_{0}^{2}} \int d^{2} x F_{\mu \nu}^{2}-\sum_{k=1}^{4} \int d^{2} x \bar{\chi}_{k} \sigma_{\mu}\left(\partial_{\mu}+i A_{\mu}\right) \chi_{k}-\sum_{k=1}^{4} \int d^{2} x \bar{\psi}_{k} \sigma_{\mu}^{*}\left(\partial_{\mu}+i A_{\mu}\right) \psi_{k}
$$

At zero topology the result of integrating out the fermions on a torus is the the same as in the 11112 model. The vector-like model also has a 't Hooft vertex $V_{v}$ which gets an expectation value in the single instanton sector.

$$
<V_{v}>\equiv \frac{\pi^{2}}{e_{0}^{4}}<\prod_{k}\left(\bar{\psi}_{k} \chi_{k}\right)>=\frac{e^{4 \gamma}}{(2 \pi)^{4}} \approx .00645
$$

Using the same regularization scheme for both models one expects that the Thirring term here $\left(\sum_{k}\left(\bar{\chi}_{k} \chi_{k}\right) \sum_{k}\left(\bar{\psi}_{k} \psi_{k}\right)\right)$ will have an induced coefficient of similar size to the one in the chiral version. In the vector-like case, depending on the sign of the Thirring coupling, the vertex would either diverge or vanish in the continuum limit. By tuning a parameter in the regularization one can arrange that an apparent limit is obtained as continuum is approached. Moreover, one can see whether the correct numerical value is indeed obtained. (Actually, one uses the bare $e_{0}$ to extract the dimensions and this might be the source of a systematic error. If measurements turn out close enough to the exact number one hopes one can ignore this error, at least at the present level of accuracy.)

Our numerical technique, the overlap, has been developed over several years starting with [9] and being finalized in [10]. We added several new tricks to increase the efficiency of the simulation. In the overlap one computes two ground states for each fermion multiplet, one corresponding to a positive mass $m_{1}$, and the other to a negative mass $m_{2}$. Previously 
$[4,7,8]$ we took $m_{1}=-m_{2}$, but the positive mass can be made infinite without spoiling the construction. At infinite mass the needed ground state is known analytically and overall computation time gets reduced by about one third. $m_{2}=-m$ is restricted to a bounded range and shall be tuned (see later) to diminish the influence of induced Thirring couplings. To be sure, $m$ stays of the order of $1 / a$, where $a$ is the lattice spacing, so the tuning really applies to ma. Another trick was already used in [4] where the gauge fields were generated using a non-compact action (the fermions always see compact fields, so this is just a technicality). We create the instantons "by hand" and adjust the measured vertex expectation value by the (analytically known) probability for the gauge action to spontaneously generate an instanton. Since instanton creation would be a Poisson process if left to the stochastic dynamics, a large statistical error would be induced (measuring the probability to produce an instanton), so the saving in computer time is substantial, by a factor of 10 roughly. A third trick we employed was to generate the constant part of the vector potentials by a weight equal to the fermion induced action in the continuum. This gave us a reduction by a factor of about 3 in computer time. A fourth trick we used was to note that charge 2 fermions "need" a finer lattice than charge 1 fermions. Thus, our charge 2 fermions were living on a lattice of size $L \times L$ while the charge 1 fermions were living on an embedded lattice of size $L / 2 \times L / 2$. (Both types of fermions interact with the same gauge field, only one uses longer paths for parallel transport on the coarser lattice.) Since there are four charge one fermions which have to be dealt with separately as they obey different boundary conditions, the time reduction is large, however it comes at the expense of some systematics. A fifth trick we employed was related to the appearance of a derivative in the chiral vertex. We extracted the analytical correction coming from the finite difference representation of the derivative of $\eta$, and we scaled the measured numbers to compensate for this effect. Finally, to ameliorate possible $S U(4)$ breaking effects induced by the boundary conditions we averaged over all index distributions in the point-split, gauge invariant, lattice vertex. We ended up using about one month of computer time on a dedicated workstation to produce the numerical results below.

We started by measuring $\left\langle V_{v}\right\rangle$ in the four flavor Schwinger model. We found that picking $m=.5$ seemed to make Thirring effects undetectable. Throughout the simulation we kept the physical size of the torus fixed, adjusting the bare gauge coupling when the lattice size is increased (i.e. when the lattice spacing is reduced). We chose $\frac{e_{0} l}{\sqrt{\pi}}=1.5$ which corresponds to the volume size in our simulation of the single flavor Schwinger model [8] (all our tori had equal sides of size $l$ ). Analytical calculations in the continuum [11] show that finite size effects reduce $\left\langle V_{v}\right\rangle$, equal to .00645 in infinite volume, to .0031 at $\frac{e_{0} l}{\sqrt{\pi}}=1.5$.

The basic method of measurement is the same as in [8], except diagonalization is now done by Householder's method rather than Jacobi's. The approach to the continuum limit can be seen from Table 1. Quite early, a smooth monotonic behavior sets in which seems 
to asymptote to the correct theoretical value within errors. Note that the sizes we quote are for a lattice which has charge one fermions at each site, so one could argue that these sizes should be doubled when comparing with results in the chiral case.

\begin{tabular}{|r|r|}
\hline$L$ & $<V_{v}>$ \\
\hline 10 & $0.0022(2)$ \\
12 & $0.0026(2)$ \\
14 & $0.0025(2)$ \\
16 & $0.0026(2)$ \\
18 & $0.0028(2)$ \\
\hline$\infty$ & 0.0031 \\
\hline
\end{tabular}

Table 1: $\left\langle V_{v}\right\rangle$ for various lattice spacings $\left(\frac{l}{L}\right)$ at $e_{0} l / \sqrt{\pi}=1.5$. At $e_{0} l / \sqrt{\pi}=\infty$ the continuum value is .00645 .

Our experience in the vector-like model led us to choose $m=.5$ also in the chiral simulation. The infinite volume continuum value for the measured vertex in the 11112 model is .081, but a finite volume computation in the continuum is not available yet. We can only guess that a similar reduction, by a factor of about $\frac{.00645}{.0031}=2.1$ will occur in our finite system.

Our results, corrected for point-split effects, are listed in Table 2. The non-negligible point-split correction factor also appears in Table 2. All in all the numbers we measure seem to be very reasonable and compatible with what we know about the exact result. We guess that the continuum limit of the vertex in the chiral case, taking into account the finite size corrections, is $\frac{.081}{2.1}=.039$. For the lattice spacings that the chiral model was simulated at, the vector model shows finite cutoff effects that diminish the vertex value by $20 \%-30 \%$. This is entirely compatible with our measurements in the chiral case. In summary, the indication is that the overlap works quantitatively in the chiral 11112 model just as well as it does in the four flavor vector model.

We feel that our simulations provide sufficient evidence to justify the investment of resources needed to carry out a large scale simulation which would seek to work in significantly larger volumes and measure the spectrum directly. It is conceivable that further savings in computer time could be obtained by "improving" the lattice Hamiltonians used to define the overlap. 


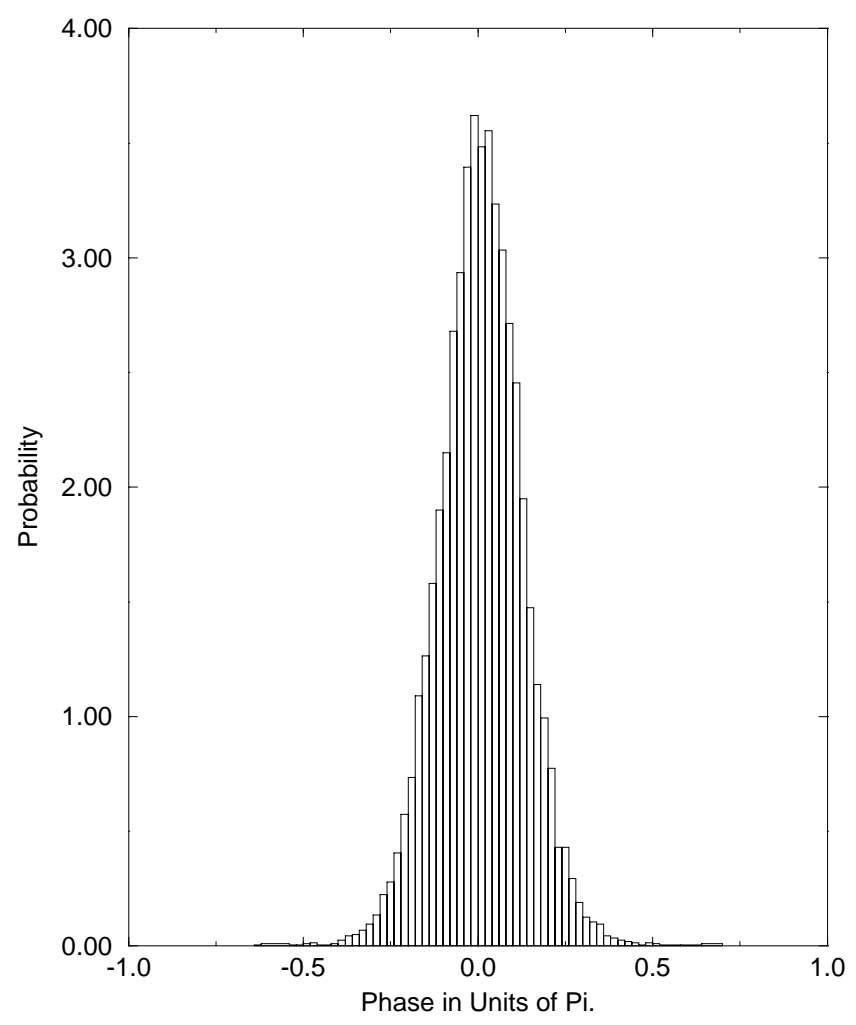

Figure 1 Phase distribution along a generic orbit carrying zero topological charge. The horizontal axis is in units of $\pi$. The phase is measured relative to the Landau gauge phase and, within errors, the average cancels the Landau phase leaving an almost real answer. The histogram contains 10,000 points.

\begin{tabular}{|r|r|r|r|}
\hline$L$ & $\langle V\rangle_{\text {Landau-gauge }}$ & $\langle V\rangle_{\text {gauge-average }}$ & point-split factor \\
\hline 8 & $0.0251(06)$ & $0.0257(08)$ & 0.6610 \\
10 & $0.0256(07)$ & $0.0264(08)$ & 0.7397 \\
12 & $0.0288(11)$ & $0.0302(15)$ & 0.7940 \\
14 & $0.0305(13)$ & $0.0317(14)$ & 0.8328 \\
16 & $0.0316(12)$ & $0.0333(14)$ & 0.8615 \\
\hline
\end{tabular}

Table 2: $\langle V\rangle$ for various lattice spacings $\left(\frac{l}{L}\right)$ at $e_{0} l / \sqrt{\pi}=1.5$. At $e_{0} l / \sqrt{\pi}=\infty$ the continuum value is .081 .

Since one of the new ingredients in the chiral case (and a very time consuming one) 


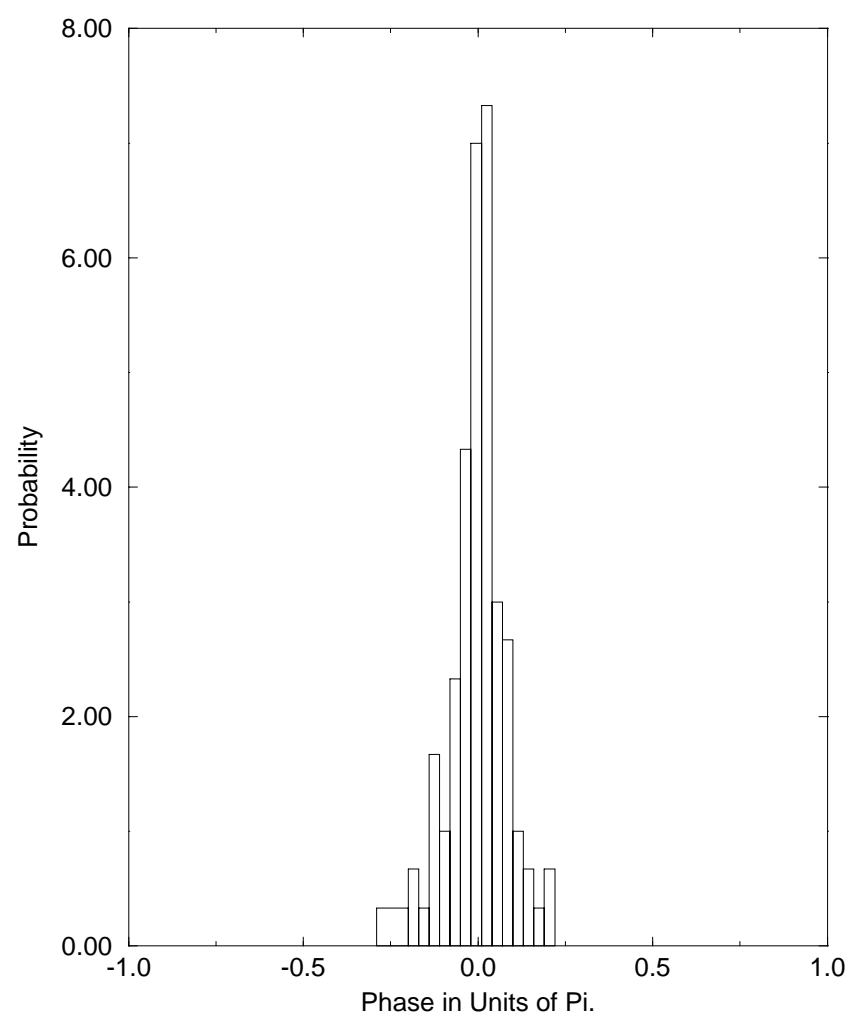

Figure 2 Phase distribution along a generic orbit carrying unit topological charge. The horizontal axis is in units of $\pi$. The phase is measured relative to the Landau gauge phase and, within errors, the average cancels the Landau phase leaving an almost real answer. The histogram contains 100 points.

is the need to do gauge averaging we show in Figures 1 and 2 two examples of the phase fluctuations in the chiral observable, one at zero topology and the other in a background carrying unit topological charge. Both examples are in statistically generic gauge field backgrounds. We have explained in $[4,10]$ that the gauge averaging should produce no net phase and should have only an irrelevant (barring Thirring terms) effect. In Table 2 we therefore show results both in a fixed (Landau like) gauge (no gauge averaging) and including gauge averaging. There is no evidence that a discernible difference will be maintained in the continuum limit between the gauge fixed and gauge averaged result. The phase distributions are quite feature-less, looking like periodic Gaussians, further indicating that indeed gauge invariance is restored without unwanted side effects (in [4] nontrivial topology was not analyzed - we now see that gauge averaging works at least as 
well in the topological charge one sector).

In addition to carrying out a large scale simulation of the two models there are some less demanding directions for future work: One should complete the solution of the Abelian model by finding exact expressions for 't Hooft's vertex on a finite torus and also include the effects of a Thirring term. In the same context it might be interesting to compute explicitly the form factors of the massless composites, as seen, for example, by a small, slowly varying external gauge field coupling to their $S U(4)$ charges. In the non-Abelian model one should examine further the possibility to obtain some exact results about its massless content. Also, there should exist some simple finite size dependent measurements that could count efficiently the number of massless particle types in either model.

\section{Acknowledgments}

This research was supported in part by the DOE under grants \# DE-FG06-91ER40614 (RN), \# DE-FG06-90ER40561 (RN) and \# DE-FG05-96ER40559 (HN). One of us (R.N.) would like to thank D.B. Kaplan for discussions.

\section{References}

[1] G. 't Hooft, Lectures at Cargese Summer Institute (Sept. 1979).

[2] S. Dimopoulos, S. Raby, L. Susskind, Nucl. Phys. B173 (1980) 208; S. Raby, S. Dimopoulos, L. Susskind, Nucl. Phys. B169 (1980) 373.

[3] T. Banks, Y. Frishman, S. Yankielowicz, Nucl. Phys. B191 (1981) 493.

[4] R. Narayanan, H. Neuberger, Nucl. Phys. B, in print, hep-lat \# 9607081.

[5] For a more detailed review see section 10 in [10].

[6] S. Coleman, "The Uses of Instantons", Erice Subnucl. 1977:805.

[7] R. Narayanan, H. Neuberger, P. Vranas, Nucl. Phys. B (Proc. Suppl.) 47 (1996) 596.

[8] R. Narayanan, H. Neuberger, P. Vranas, Phys. Lett. B353 (1995) 507.

[9] R. Narayanan, H. Neuberger, Phys. Lett. B302 (1993) 62.

[10] R. Narayanan, H. Neuberger, Nucl. Phys. B443 (1995) 305.

[11] This requires a trivial generalization to several flavors of the work in I. Sachs, A. Wipf, Helv. Phys. Acta 65 (1992) 652. 